Rinaldo Bellomo

\title{
The avoidable death of a boy and the relentless pursuit for evidence
}

Received: 22 June 2013

Accepted: 29 June 2013

Published online: 16 July 2013

(C) Springer-Verlag Berlin Heidelberg and ESICM 2013

R. Bellomo (৫)

Australian and New Zealand Intensive Care Research Centre and Department of Epidemiology and Preventive Medicine,

Monash University, Melbourne, VIC, Australia

e-mail: rinaldo.bellomo@austin.org.au

Tel.: +61-3-94965992

Fax: +61-3-94963932

"In dwelling, live close to the ground.

In thinking, keep to the simple.

In conflict, be fair and generous.

In governing, do not try to control.

In work, do what you enjoy.

In family life, be completely present."

Lao Tzu (Tao Te Ching)

I remember it like yesterday, but it was 25 years ago. I was a young doctor taking my first steps in clinical research. I was working as a nephrology fellow, and for the previous year I had been working on increasing our understanding of the physiological and biological effects of continuous veno-venous hemodiafiltration (CVVHDF). It was Friday night, and a 16-year-old boy was transferred from the intensive care unit (ICU) of a large peripheral hospital with multiorgan failure following trauma. He had uncontrolled uremia. He arrived (as is so often the case) at approximately $8 \mathrm{pm}$. I remember discussing the renal management plan with the ICU team, as I was going to be away for the weekend. We would stabilize the patient overnight, continue current treatment, and then begin CVVHDF in the morning. This would gently decrease his urea without any clinically important solute shifts or effects on cerebral edema. Intermittent hemodialysis as a means of controlling uremia in this patient was unsafe. I left the ICU and was away for the weekend. When I returned on Monday, I went straight to the ICU, bed 1, to see how this young patient had hopefully improved over the weekend. The boy was no longer there. He had died. The nephrology specialist on duty for the weekend had decided to countermand the plan. He prescribed dialysis. Within 20 min of the start of dialysis, the boy's pupils became fixed and dilated and he died. I can still remember the emotions: a mixture of anger, frustration, indignation, and sadness. I felt this boy had been killed by the reckless use of dialysis. I have spent the last 25 years in the relentless pursuit of all the evidence that I could obtain to show clinicians what I felt I could see and what I felt so often they could not see or accept in this field of practice. It has been a complex and sometimes frustrating journey, and it has led me away from my initial plans to become a nephrologist to a now long career in critical care medicine instead. It has spawned many studies and presentations, and, over time, the use of continuous renal replacement therapy (CRRT) has grown from a few centers to the whole of Australia and then to many other countries to finally being the dominant form of acute renal replacement therapy in the world. The changes have taken a long time, much longer than I had hoped at that time, and are not yet complete but have, nonetheless, been important and gratifying. Still, in my mind, much remains to be done as intermittent dialysis continues to adversely affect renal recovery. However, this journey has affected my other professional choices profoundly over the last 20 years. The pursuit of evidence in the field of acute kidney injury and the growing realization that practice change worldwide would only come from randomized controlled trials have eventually changed my passion and 
commitment to the broader agenda of conducting and completing large randomized controlled trials. This relentless commitment has helped me see that the process of evidence-gathering must include all aspects of evidence, from clinical observation at the bedside to animal experimentation, from systematic and reflective review and analysis of the literature to the careful rereading of classics in intensive care medicine and physiology, from discussions with colleagues to listening to nurses, from debates with other investigators to listening to patients and families, from careful observation of practice and epidemiology to pilot work in humans, from feasibility and safety randomized controlled studies to phase III multicenter pivotal trials. The desire to stop patients and families from being victims of "bad medicine," like the boy so long ago, has more recently taken my work outside the ICU, where I think the new frontier of our practice now lies: rapid response teams and endof-life care. Increasingly, much of my clinical time is spent with families to explain and to understand when we should escalate and when we should aim for the comfort, care, and dignity of a good death. More and more I believe that compassion and care matter as much as, if not more than, our often deluded beliefs in concepts such as efficacy, physiological gain, and biological manipulation. I preach the 5 C's: compassion, care, communication, competence, and collegiality. Compared with 30 years ago, I worry much, much more about safety than efficacy. I cannot know whether I am right or wrong. Time will tell. However, as I approach my own death, whatever I do, wherever I speak, whatever research project I work on, whatever paper I write, I still remember the young boy who needed CRRT, got dialysis instead, and died.

Beyond this abiding memory, for ICUs and hospitals the world over, there is still so much to be done to improve safety. I feel it with an unending and unchanged passion within my heart, even as I age and my physical energy is diminished.

Yet, as I age and my stamina wanes, I am comforted by Tennyson's words:

"Though much is taken, much abides; and though

We are not now that strength which in old days

Moved earth and heaven, that which we are, we are...

Made weak by time and fate, but strong in will

To strive, to seek, to find, and not to yield."

And when I return to work each morning to face an endless agenda of research and clinical commitments, I remember the words of Robert Frost, which have been resonating in my heart and mind since I first read them at 17 years of age “...The woods are lovely, dark and deep, but I have promises to keep, and miles to go before I sleep, and miles to go before I sleep."

Conflicts of interest The author declares that there are no conflicts of interest in relation to this manuscript. 